\title{
Diferença de Letalidade Hospitalar do Infarto Agudo do Miocárdio entre Homens e Mulheres submetidos a Angioplastia Primária
}

\author{
Luiz Carlos Santana Passos, Antonio Alberto Lopes, Fábio Peroba Esteves, \\ Fabiano Marins de Oliveira Santos
}

Salvador, BA

Objetivo - Comparar a letalidade hospitalar $(\mathrm{LH})$ de homens e mulheres submetidos a angioplastia transluminal coronária primária (ATCP) como estratégia de reperfusão no infarto agudo do miocárdio (IAM).

Métodos - Estudo de coorte retrospectivo baseado em informações de prontuários médicos de pacientes hospitalizados devido a IAM em hospital de referência para doença coronária, utilizando preferencialmente a ATCP como técnica de reperfusão miocárdica precoce. Foram incluidos 83 pacientes, 35 (42\%) mulheres e 48 (58\%) homens, que preencheram critérios para reperfusão miocárdica precoce.

Resultados - $A$ média de idade das mulheres foi superior a dos homens (66 \pm 10 vs $58 \pm 11$ anos; $p<0,001)$. As mulheres também apresentaram uma maior freqüência de diabetes mellitus $(37,1 \%$ vs 6,4\%; $p<0,001)$. Apesar destas diferenças na idade e na freqüencia de diabetes mellitus, os percentuais de pacientes em que a ATCP foi considerada sucesso foram similares entre homens (83,3\%) e mulheres (82,3\%). A LH foi também bastante semelhante entre homens e mulheres; $14,6 \%$ vs $14,3 \%$, respectivamente.

Conclusão - A possibilidade de que o excesso de risco de morrer entre as mulheres com IAM em relação aos homens possa ser reduzido ou até eliminado com o uso da ATCP apóia a necessidade de que se desenvolva um ensaio clínico a fim de avaliar esta questão.

Palavras-chave: infarto agudo do miocárdio, letalidade hospitalar e angioplastia coronária primária

\section{Difference in the Case-Fatality Rate of Acute Myocardial Infarction between Men and Women Submitted to Primary Angioplasty}

Purpose - To compare the in-hospital case-fatality rate of men and women submitted to percutaneous transluminal coronary angioplasty (PTCA) as a strategy of reperfusion in acute myocardial infarction (MI).

Methods - A retrospective cohort study based on information abstracted from medical records of $M I$ patients admitted to a hospital where PTCA is used as the prefered method for early myocardial reperfusion. A total of 83 patients, 35 (42\%) women and 48 (58\%) men, who met the criteria for early myocardial reperfusion was included in the analysis.

Results - The mean age of women was higher than that of men ( $66 \pm 10 \mathrm{vs} 58 \pm 11$ years; $p<0.001)$. The women also had a higher frequency of diabetes mellitus (37.1\% vs $6.4 \% ; p<0.001)$. Despite these differences in age and in the frequency of diabetes mellitus, the percentage of patients in whom the PTCA was considered successful was similar between men (83.3\%) and women (82.3\%). The case-fatality rate was also similar between men and women; $14.6 \%$ and $14.3 \%$, respectively.

Conclusion - The possibility that the excess risk of death in women with MI may be reduced and even eliminated with the use of PTCA, supports to the need for the development of a clinical trial to assess this question.

Keywords: acute myocardial infarction, case-fatality rate, primary coronary angioplasty

Arq Bras Cardiol, volume $71\left(n^{0} 4\right), 587-590,1998$

Hospital Português e Faculdade de Medicina da Universidade Federal da Bahia Correspondência: Luiz Carlos Santana Passos - Rua Amir Macedo, 119/1003 40285-460 - Salvador, BA

Recebido para publicação em 6/3/98

Aceito em 22/7/98
Diversos trabalhos mostram, de forma consistente, que a letalidade hospitalar ( $\mathrm{LH})$ do infarto agudo do miocárdio (IAM) é maior entre as mulheres do que entre os homens ${ }^{1-9}$. As razões para essa diferença de letalidade do IAM entre os sexos, no entanto, não são completamente 
entendidas. Inicialmente, a maior LH do IAM entre as mulheres foi considerada como decorrente da idade mais avançada no momento do infarto ${ }^{3,4}$. Porém, vários estudos mostraram que o efeito da idade mais avançada é insuficiente para justificar o pior prognóstico na fase hospitalar do IAM entre as mulheres ${ }^{6,7}$. Evidências de trabalhos mais recentes apóiam a possibilidade de que fatores mais relacionados com o sexo feminino, como menor acesso a cuidados médicos, maior intervalo entre o inicio de sintomas, intervenções terapêuticas para o IAM e maior freqüência de comorbidades associadas podem também interferir nas diferenças de prognóstico entre homens e mulheres com IAM ${ }^{8,9}$.

As primeiras observações sobre o pior prognóstico do IAM entre as mulheres foram relatadas na década de 70 e essas diferenças entre os sexos não foram modificadas pela introdução da terapia de reperfusão baseada na trombólíse química $^{7,10}$. Em relação ao uso da angioplastia transluminal coronária primária (ATCP), existem evidências de que esta técnica é mais eficaz do que a trombólise em promover a patência arterial ${ }^{11-13}$. Além disso, a ATCP pode contornar o problema da menor elegibilidade das mulheres para terapia trombolítica, uma vez que a ATCP pode ser utilizada em praticamente todos os pacientes com IAM. Recentemente, Stone e col, numa análise secundária do PAMI trial, observou que a ATCP reduz mais acentuadamente a LH do IAM em mulheres do que em homens ${ }^{14}$. Estes achados de Stone e col e as evidências de que a ATCP é superior à trombólise como método de reperfusão motivou a realização do presente estudo, no sentido de avaliar se a relação entre uso de ATCP e redução da diferença de LH entre homens e mulheres com IAM é um fato generalizado para outros serviços.

\section{Métodos}

Foram revistos retrospectivamente todos os prontuários de pacientes admitidos na Unidade Coronariana do Hospital Português, Salvador-BA com suspeita diagnóstica de IAM, entre fevereiro/92 e dezembro/96. Após a identificação dos pacientes com IAM, selecionou-se para análise aqueles submetidos à angioplastia primária que apresentavam os seguintes critérios para utilização de terapias de reperfusão: 1) dor torácica compatível com IAM com duração >30min e não responsiva ao uso de nitrato sublingual; 2) tempo máximo entre o início dos sintomas e admissão hospitalar $\leq 12 \mathrm{~h}$; 3) supradesnível do segmento ST >1mmem pelo menos duas derivações da mesma parede ou bloqueio completo de ramo esquerdo no momento da admissão. A direção da investigação foi a seleção dos pacientes na admissão para os eventos, caracterizando desta forma um estudo de coorte. Sexo constituiu-se na principal variável preditora e, morte durante o internamento no evento de maior interesse.

As variáveis clínico-demográficas dos pacientes foram definidas tomando por base os dados colhidos no momento da admissão hospitalar. Para o diagnóstico eletrocardiográfico (ECG) de infarto foram considerados os laudos do ECG registrados em prontuário. Todos os pacientes realizaram dosagens seriadas de creatinofosfoquinase (CK total) e da fração miocárdica desta enzima (CK-MB). Para o diagnóstico de IAM, consideraram-se apenas aqueles pacientes com elevação superior a duas vezes o valor máximo admitido normal (10 ui). Os diagnósticos de diabetes mellitus (DM) e hipertensão arterial foram estabelecidos de acordo com dados de história pregressa ou diagnóstico durante o internamento. O diagnóstico de infarto prévio foi baseado na história clínica e evidências eletrocardiográficas. A ausência da anotação de um determinado dado considerado obrigatório de ser mencionado, quando presente, (ex. história pregressa ou seqüela de acidente vascular cerebral) foi avaliada como não ocorrência da condição. Dados vitais (pressão arterial, freqüência cardíaca e freqüência respiratória) e classificação de Killip foram obtidos através do exame físico da admissão. Em relação ao uso de fármacos foram consideradas as prescrições médicas de agentes utilizados por pelo menos $48 \mathrm{~h}$.

Os testes estatísticos (teste exato de Fisher, para diferenças entre proporções e teste $\boldsymbol{t}$ de Student, para diferenças de médias) foram utilizados para comparar homens e mulheres no que diz respeito aos seus perfis no momento da admissão, possíveis diferenças em abordagens terapêuticas durante o período hospitalar e eventos clínicos. Os testes estatísticos foram realizados através do software Epi-Info ${ }^{15}$. O intervalo de confiança (IC) de 95\% do risco relativo(RR) foi determinado através do software STATA, versão $5^{16}$.

\section{Resultados}

Foram identificados 176 casos que preenchiam os critérios para terapia de reperfusão miocárdica na fase aguda do IAM, sendo que 83 pacientes, 48 (58\%) homens e 35 (42\%) mulheres, foram submetidos a ATCP (tab. I). As mu-

\begin{tabular}{|c|c|c|c|}
\hline \multirow[b]{2}{*}{ Característica } & \multicolumn{2}{|c|}{ Sexo } & \multirow[b]{2}{*}{$\mathrm{p}$} \\
\hline & Feminino & Masculino & \\
\hline & $\mathrm{n}=35(42 \%)$ & $\mathrm{n}=48(58 \%)$ & \\
\hline \multicolumn{4}{|l|}{ Idade } \\
\hline média (anos) & $66,0 \pm 10$ & $59,7 \pm 11$ & $<0,001$ \\
\hline mediana (anos) & 68 & 59 & \\
\hline \multicolumn{4}{|l|}{ Tempo de sintomas } \\
\hline média (horas) & $4,8 \pm 2,7 \mathrm{~h}$ & $4,6 \pm 3,7 \mathrm{~h}$ & 0,40 \\
\hline mediana (horas) & 4 & 3 & \\
\hline Hipertensão arterial & $62,9 \%$ & $56,3 \%$ & 0,54 \\
\hline Diabetes mellitus & $37,1 \%$ & $6,4 \%$ & $<0,001$ \\
\hline \multicolumn{4}{|l|}{ Classe de Killip } \\
\hline I & $65,7 \%$ & $79,2 \%$ & 0,17 \\
\hline$>\mathrm{I}$ & $34,3 \%$ & $20,8 \%$ & \\
\hline \multicolumn{4}{|l|}{ Localização do infarto } \\
\hline Anterior & $60,0 \%$ & $47,9 \%$ & 0,27 \\
\hline \multicolumn{4}{|l|}{ Terapias farmacológicas } \\
\hline Ácido acetilsalicílico & $94,3 \%$ & $97,9 \%$ & 0,39 \\
\hline Betabloqueadores & $57,1 \%$ & $83,0 \%$ & 0,01 \\
\hline Bloqueadores canais $\mathrm{CA}^{++}$ & $2,9 \%$ & $4,3 \%$ & 0,73 \\
\hline Inibidores da ECA & $48,6 \%$ & $29,9 \%$ & 0,08 \\
\hline Heparina & $85,7 \%$ & $93,6 \%$ & 0,23 \\
\hline
\end{tabular}




\begin{tabular}{|c|c|c|c|c|c|}
\hline & \multicolumn{2}{|c|}{ Morte } & \multirow[b]{2}{*}{ Letalidade } & \multirow{2}{*}{$\begin{array}{c}\mathrm{RR} \\
(\mathrm{IC} 95 \%) \\
\end{array}$} & \multirow[b]{2}{*}{ valor de $p$} \\
\hline & Sim & Não & & & \\
\hline Feminino & 5 & 30 & $14,3 \%$ & $0,98(0,48-2,03)$ & 1,00 \\
\hline Masculino & 7 & 41 & $14,6 \%$ & & \\
\hline
\end{tabular}

lheres eram em média 6 anos mais idosas do que os homens, no momento da admissão hospitalar (66,0 vs 59,7 anos), apresentavam maior frequiência de $\operatorname{DM}(34,2$ vs $10,9 \%)$ e receberam menos freqüentemente agentes betabloqueadores $(56,2$ vs $81,0 \%)$.

Quando a artéria relacionada ao infarto apresentou fluxo TIMI II ou III e lesão residual inferior a 50\% após a ATCP, o resultado do procedimento foi considerado sucesso. Isto ocorreu em $69(83,1 \%)$ pacientes, não havendo diferenças em relação ao sexo $(83,3 \%$ para homens e $82,3 \%$ para as mulheres).

Na tabela II é mostrado que a LH do IAM entre os pacientes submetidos a ATCP foi bastante semelhante entre homens $(14,3 \%)$ e mulheres $(14,6 \%)$, correspondendo a um RRde 0,98 (IC 95\% = 0,48-2,03).

\section{Discussão}

Confirmando dados da literatura ${ }^{14,17}$, no presente estudo a LH do IAM foi semelhante entre homens e mulheres tratados com ATCP. Este achado deve ser visto como significativo do ponto de vista clínico e de saúde pública, considerando-se que a grande diferença de mortalidade (maior em mulheres) devido ao IAM, entre homens e mulheres, vem se mantendo constante ao longo dos anos, mesmo após a introdução de terapia trombolítica ${ }^{7,10,18}$. Numa revisão sistemática, envolvendo 55.000 pacientes que participaram de ensaios clínicos ${ }^{10}$, o risco de morte foi aproximadamente $60 \%$ maior em mulheres do que em homens, independente do uso de fibrinolíticos ou placebo. É importante observar que apesar da trombólise não reduzir a diferença de mortalidade entre os sexos, as evidências indicam que esta terapia é igualmente benéfica em homens e mulheres.

Embora os resultados do presente trabalho sejam entusiasmantes no sentido de apontar para uma alternativa de redução da diferença do IAM entre os sexos, a falta de um grupo de comparação tratado apenas com trombolíticos e o tamanho ainda relativamente pequeno da amostra devem ser vistos como limitações para conclusões mais definitivas. Alguns pontos, no entanto, merecem ser comentados: $\left.1^{\circ}\right)$ a trombólise química, apesar de se constituir em um avanço na terapêutica do IAM, resulta em reperfusão miocárdica ótima (i.e., obtenção de fluxo com perviabilidade TIMI III) em, no máximo, $55 \%$ dos pacientes ${ }^{19}$. Por outro lado, a ATCP permite atingir perviabilidade TIMI III em até $90 \%$ dos pacientes ${ }^{20} ; 2^{\circ}$ ), tem sido mostrado que as mulhe- res apresentam maior risco de hemorragia cerebral com os agentes trombolíticos atualmente utilizados, o que constitui num fator adicional de mortalidade; $3^{\circ}$ ) a cinecoronariografia no momento da admissão permite que pacientes com doença coronária obstrutiva grave e anatomia desfavorável para angioplastia possam ser submetidos a tratamento cirúrgico precoce, evitando desta forma mortes ainda na fase hospitalar. Finalmente, deve ser observado que a ATCP, diferente da trombólise, pode ser utilizada em quase todos os pacientes com IAM. Estas vantagens da ATCP (em relação a trombólise) devem ser vistas como potenciais explicações para o seu efeito na redução da diferença de LHentre os sexos.

A consistência dos achados do presente trabalho com os resultados de dois estudos anteriores reduz a possibilidade de que as semelhanças de LH do IAM entre homens e mulheres sejam totalmente explicadas por erro tipo II devido ao tamanho relativamente pequeno da amostra. Utilizando uma amostra bem maior do que a do presente estudo, Vacek e $\operatorname{col}^{17}$, também observaram importante redução da diferença de letalidade do IAM entre homens e mulheres tratados com ATCP, porém o estudo apresenta também a limitação da falta de um grupo de comparação, tratado com trombólise ou outras modalidades terapêuticas.

Diferente do presente estudo e do de Vacek e col, o de Stones e col ${ }^{14}$ inclui um grupo de comparação constituído de pacientes submetidos a trombólise com r-tpa, salientando que no grupo de pacientes submetidos a trombólise com r-tpa o risco de morrer foi 10,5 pontos percentuais maior nas mulheres em relação aos homens. Por outro lado, no grupo de pacientes submetidos a ATCP essa diferença entre os sexos foi bem menor, 1,9 pontos percentuais, apesar de que as mulheres incluídas no PAMI trial, semelhantes as do presente estudo, apresentaram maior média de idade e maior frequiência de DM. A crítica que se pode fazer ao estudo de Stones e col é que o mesmo não foi primariamente desenhado para avaliar os efeitos de diferentes tipos de tratamento em reduzir as diferenças de mortalidade entre os sexos, sendo portanto uma análise secundária dos dados do PAMI trial $^{20}$. Consideradas as limitações, o presente estudo e os anteriores podem ser vistos como evidências ainda não definitivas, de que a ATCP é mais eficaz em reduzir a $\mathrm{LH}$ do IAM nas mulheres do que nos homens.

Resumindo, há evidências apoiando a possibilidade de que o excesso de risco de morrer, observado entre as mulheres em relação aos homens desde a era pré-trombólise, possa ser reduzido ou até eliminado pelo uso da ATCP. 
É possível que esse maior efeito da ATCP nas mulheres esteja relacionado com a maior taxa de perviabilidade arterial com fluxo TIMI III, menor incidência de hemorragia cerebral e indicação mais precoce de tratamento cirúrgico em pacientes com anatomia desfavorável para angioplastia por balão.
Os resultados do presente estudo apóiam a necessidade de que se implemente um ensaio clínico desenhado especificamente para esclarecer de modo mais definitivo o papel da ATCP na redução da diferença de mortalidade entre os homens e as mulheres.

\section{Referências}

1. Greenland P, Reicher-Reiss H, Goudbourt U, Behar S, Israeli - SPRINT investigators. In-hospital and 1-year mortality in 1.524 women after myocardial infarction. Circulation 1991; 83: 484-91.

2. Fiebach NH, Viscoli CM, Horwitz RJ - Differences between women and men in survival after myocardial infarction: biology or methodology ? JAMA 1990; 263: 1092-6.

3. Dittrich H, Gilpin E, Nicod P, Cali G, Henning H, Ross J - Acute myocardial infarction in women:influence of gender on mortality and prognostic variables. Am J Cardiol 1988; 62: 1-7.

4. Maynard C, Litwin PE, Martin JS, Weaver WD - Gender differences in the treatment and outcomes of acute myocardial infaction-results from the Myocardial Infartion Triage and Intervention Registry. Arch Itern Med 1992; 152: 972-6.

5. Passos LC, Lopes AA, Souza CAM et al - Preditores de mortalidade hospitalar na era trombolítica para o infarto agudo do miocárdio em Salvador (BA). Arq Bras Cardiol 1997; 68: 249-55.

6. Puletti M, Sunseri L, Curione M, Erba SM, Borgia C - Acute Myocardial Infarction: sex-related differences in prognosis. Am Heart J 1984; 108: 63-6.

7. Weaver WD, Harvey DW, Wilcox RG et - Gusto Investigators. Comparisons of caracteriscs and outcomes among women and men with acute myocardial infarction treated with thrombolytic therapy. JAMA 1996; 275: 777-83.

8. Kostis JB, Wilson A, O'Dowd K, Midas - Study Group. Sex differences in the management and long-term outcome of acute myocardial infarction. Circulation 1994; 90: 1715-32.

9. Clarke KW, Gray D, Keating NA and Hampton J - Do Women with acute myocardial infarction receive the same treatment as men. Br Med J 1994, 309: 563-8.

10. Fibrinolytic Therapy Trialists (FTT) Collaborative Group - Indications for fibrinolytic therapy in suspected acute myocardial infarction: colaborative overview of early mortality and major morbidity results from all randomised trials of more than 1000 patients. Lancet 1994; 343: 311-22.
11. Michels KB, Yusuf S - Does PTCA in acute myocardial infarction affect mortality and reinfarction rates? a quantitative overview (meta-analysis) of the randomized clinical trials. Circulation 1995; 91: 476-85.

12. Every NR, Parsons LS Hlatky M and Myocardial Infarction Triage and Intervention Investigators - A comparison of thrombolytic therapy with primary coronary angioplasty for acute myocardial infarction. N Engl J Med 1996; 335 : 1253-60.

13. Gusto IIB investigators - A clinical trial comparing primary coronary angioplasty with tissue plasminogen activator for acute myocardial infarction (Gusto IIb). N Eng J Med 1997; 336: 1621-7.

14. Stone GW, Grines CL, Browne KF and Primary Angioplasty in Myocardial Infarction Study Group - Comparison of in-hospital outcome in men versus women treated by either thrombolytic therapy or primary coronary angioplasty for acute myocardial infarction. Am J Cardiol 1995; 75: 987-92.

15. Dean AG, Dean JA, Coulombier D et al - Epi Info, version 6: - A word-processing, database, and statistics program for public health on IBM-compatible microcomputers. Centers for Disease Control and Prevention, Atlanta, 1995.

16. Stata, version 5 - Stata Corporation, College Station, TX, 1997.

17. Vacek JL, Rosamond TL, Kramer PH et al - Sex-related diferences in patients undergoing direct angioplasty for acute myocardial infarction. Am Heart J 1993; 126: 521-5.

18. Malacrida R, Genone M, Maggioni AP et al - A comparison of the early outcome of acute myocardial infarction in women and men. NEngl J Med 1998; 338: 8-14.

19. The Gusto Angiographic Investigators - The effects of tissue plasminogen activactor, streptokinase, or both on coronary artery pateny, ventricular function and survival after acute myocardial infarction. N Eng J Med 1993; 329: 1615-22.

20. Grines CL, Browne KF, Marco J and Primary Angioplasty in Myocardial Infarction Study Group - A comparison of immediate angioplasty with thrombolytic therapy for acute myocardial infarction. N Engl J Med 1993; 328: 673-8. 